POS $\quad$ PROCEEDINGS

\title{
Three different types of the gamma-ray bursts
}

\author{
Attila Mészáros \\ Astronomical Institute, Faculty of Mathematics and Physics, \\ Charles University, Czech Republic \\ E-mail: meszaros@cesnet.cz
}

\section{Zsolt Bagoly}

Eötvös University, Budapest, Hungary

E-mail: zsolteyela.elte.hu

\section{Lajos G. Balázs}

Konkoly Observatory, Budapest, Hungary

E-mail: balazs@konkoly.hu

\section{Jakub Řípa}

Astronomical Institute, Faculty of Mathematics and Physics, Charles University, Czech Republic

E-mail: ripa@sirrah.troja.mff.cuni.cz

\section{Péter Veres}

Eötvös University, Budapest, Hungary

Bolyai Military University, Budapest, Hungary

Konkoly Observatory, Budapest, Hungary

E-mail: veresp@elte.hu

\begin{abstract}
The authors have shown that there are three subclasses of the gamma-ray bursts. They can be called as short, intermediate and long ones, because they can be separated with respect to their durations. The short and long bursts are physically different phenomena - astrophysical meaning of the intermediate bursts is unclear. The short and intermediate bursts are distributed anisotropically on the sky. This behavior can have a remarkable impact on the cosmology. The purpose of this contribution is a survey of this subject.
\end{abstract}

25th Texas Symposium on Relativistic Astrophysics

December 6-10, 2010

Heidelberg, Germany 
Table 1: Comparison of the probabilities that the occurrence of three GRB groups is accidental as were gained from different methods and data samples. F-test compares the best $\chi^{2}$ fits (two and three Gaussian curves) of the $\log T_{90}$ duration histogram [2]. ML is the Maximum Likelihood ratio test applied either on the $\log T_{90}$ durations or on the $\log T_{90}-\log H$ (hardness ratio) pairs. "?" means that from the article it is not clear what the significant level is. "—" means that a test has not been done yet on the given data sample.

\begin{tabular}{lrrrr}
\hline Method & BATSE & Swift & RHESSI & BeppoSAX \\
\hline F-test $\left(T_{90}\right)$ & $<10^{-4}[2]$ & $\simeq 0.03[8]$ & $0.069[11]$ & - \\
ML $\left(T_{90}\right)$ & $5 \times 10^{-3}[4]$ & $4.6 \times 10^{-3}[9]$ & $3.6 \times 10^{-4}[11]$ & $0.037[12]$ \\
ML $\left(T_{90}\right.$ vs. $\left.H\right)$ & $<10^{-10}[5]$ & $10^{-8}[10]$ & $1.3 \times 10^{-3}[11]$ & - \\
Other methods & $\ll 10^{-4}[3]$ & - & - & - \\
Other methods & $?[6,7]$ & & & \\
\hline
\end{tabular}

\section{Introduction}

The gamma-ray bursts (GRBs) are not unitary phenomena, because they can be separated, from the observational point of view, into different subclasses. In addition, these events are in cosmological distances, and are not obscured in the Galactical plane; hence, they may well serve as an observational verfication of the isotropical distribution on the sky.

The authors published several articles during the last years from this topic. These papers are surveyed in this contribution.

\section{Three subgroups of GRBs}

It is a long known result that GRBs can be separated into two different subgroups [1]. In 1998 two independent articles appeared declaring the existence of three different subgroups of GRBs $[2,3]$. Since that time several statistical analyses on different satellite databases confirmed the existence of three subgroups. In Table 1 there is a summary of these statistical tests.

The main variable used in these tests is the so called $T_{90}$ duration, which is the time during which the cumulative counts increase from $5 \%$ to $95 \%$ [1]. The second variable is the hardness, which is the ratio of fluences (total number of detected photons during the existence of a burst) in different energy channels $[1,2]$. The choice of these channels can be different for different satellites. These three subgroups for the RHESSI database are shown on Figure 1.

Two essential notes should be added here.

First, it is not necessary that these three - from the statistical point of view significantly different - subgroups are also physically different phenomena. The short and long bursts are doubtlessly different objects [13], but for the third subgroup (intermediate in duration) the most probable candidates are the so called X-ray flashes (XRFs), which need not form an astrophysically separate class of bursts (for more details see [14]). In any case, the physical meaning of the third group is not satisfactorily explained yet. 
Figure 1: The three subgroups in the RHESSI database. Any cross denotes a GRB. $T_{90}$ is in seconds (i.e., if $\log T_{90}=0$, then $T_{90}=1 s, \ldots$ ), $H$ is the dimensionless hardness (for more details see [11]).

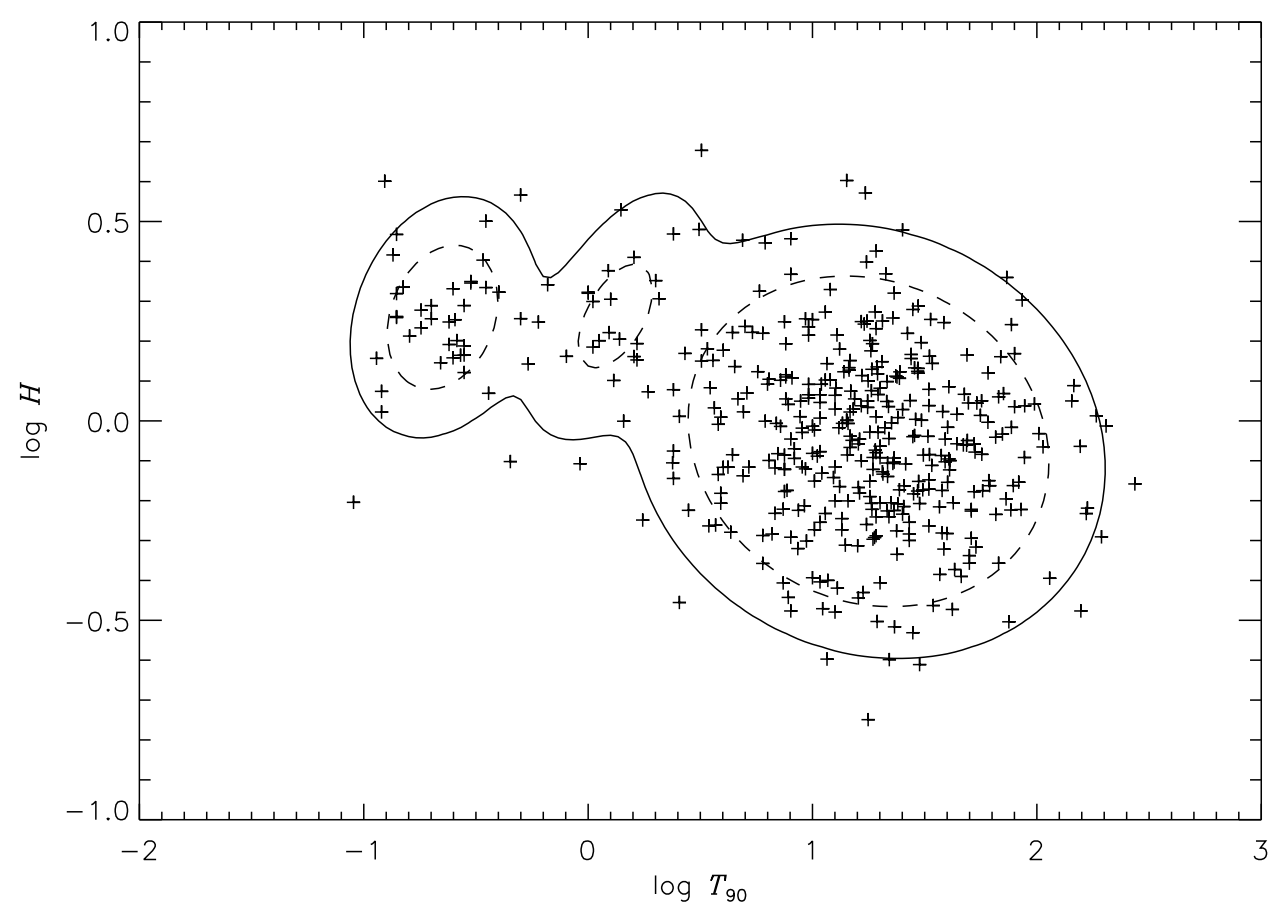

Second, in tests mentioned in Table 1, the assumption of more than three subgroups was also studied. For the BATSE database [5] a 6.2\% significance was reached. All other tests gave even weaker evidences. Hence, no statistical support for the existence of four or more subgroups was found.

\section{Anisotropy of the sky distribution of the short and intermediate subgroups of GRBs}

A decade ago the authors provided several different tests probing the intrinsic isotropy in the angular sky-distribution of GRBs collected in BATSE Catalog [15, 16, 17, 18]. Summarizing the results of these studies one may conclude: A. The long subgroup $\left(T_{90}>10 \mathrm{~s}\right)$ seems to be distributed isotropically; B. The intermediate subgroup $\left(2 s \leq T_{90} \leq 10 s\right)$ is distributed anisotropically on the $\simeq(2-3) \%$ significance level; C. For the short subgroup $\left(2 s>T_{90}\right)$ the assumption of isotropy is rejected only on the $8 \%$ significance level; D. The long and the short subclasses, respectively, are distributed differently on the $0.7 \%$ significance level.

Because mainly the situation concerning the short GRBs was unclear, a more powerful testing of the isotropy on the BATSE data was provided [19]. Three methods were used (more details 
Figure 2: The Voronoi diagram of the BATSE short bursts with $T_{90}<2 s$ and with $0.65<P_{256}<2$, where $P_{256}$ is the so called peak-flux on the $0.256 \mathrm{~s}$ scale in units photons $/\left(\mathrm{cm}^{2} s\right)$. Any dot denotes a GRB on the sky (Aitoff projection, Galactical coordinates). The polygon around a point denotes the area being nearer to this point than to any other GRB. From the character of these polygons (size, edges, angles,...) the nonrandomnesses can be deduced (for more details see [19]).

$$
\text { Short1 GRBs }\left(T_{90}<2 \text { s, } 0.65<P_{256}<2\right)
$$

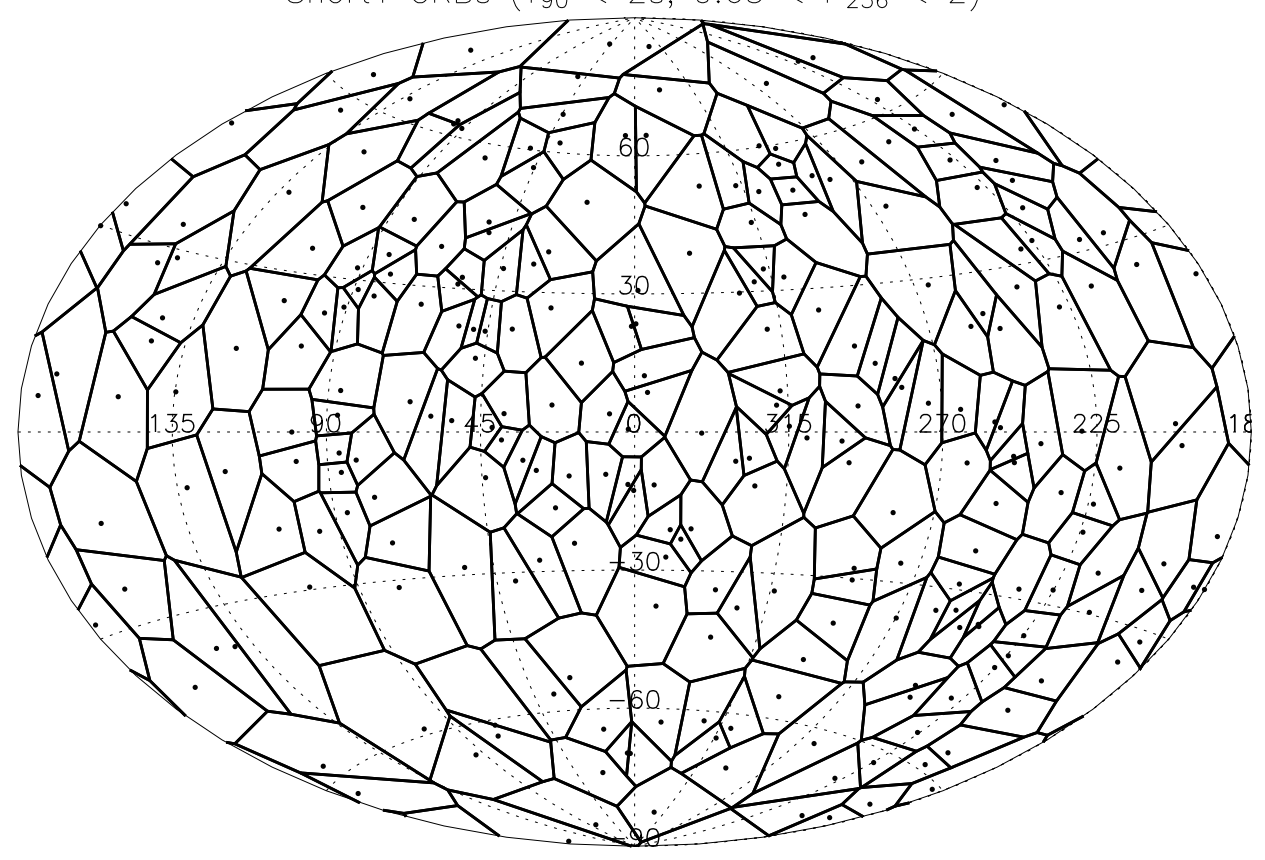

about these tests can be found in [19]). The first one is the method called "Voronoi tesselation" based on the Voronoi diagram (also known as Dirichlet tesselation or Thiessen polygons). This diagram provides a partition of the point field on a sphere. The points on sphere may be distributed completely randomly or non-randomly; the non-random distribution may have different characters (clustering, filaments, etc.). The second method is called "Minimal spanning tree". This method considers the distances (edges) among the points (vertices). Clearly, there are $N(N-1) / 2$ distances among $N$ points. A spanning tree is a system of lines connecting all the points without any loops. The third method uses the "Multifractal spectrum". The idea here is the following: Let denote $P(\varepsilon)$ the probability for finding a point in an area of $\varepsilon$ radius. If $P(\varepsilon) \propto \varepsilon^{\alpha}$, then $\alpha$ is called the local fractal dimension. Obviously, $\alpha=2$ should be for a completely random process on the sphere surface. In Figure 2 the Voronoi diagram of short BATSE GRBs is shown.

The results of these tests are decisive: The short GRBs are non-random on a $<0.1 \%$ significance level. Also the intermediate sample gave a significant deviation $(1.5 \%)$ from the full randomness in accordance with the earlier studies. The long sample remained random.

As far as known, these anisotropy tests were done only for the BATSE database. 
Figure 3: The cumulative redshift distribution of GRBs with directly measured redshifts and detected by the Swift satellite. The solid (dashed, dotted) line denotes the short (intermediate, long) GRBs. A skip means a GRB - as it is usual in the Kolmogorov-Smirnov test (for more details see [10]).

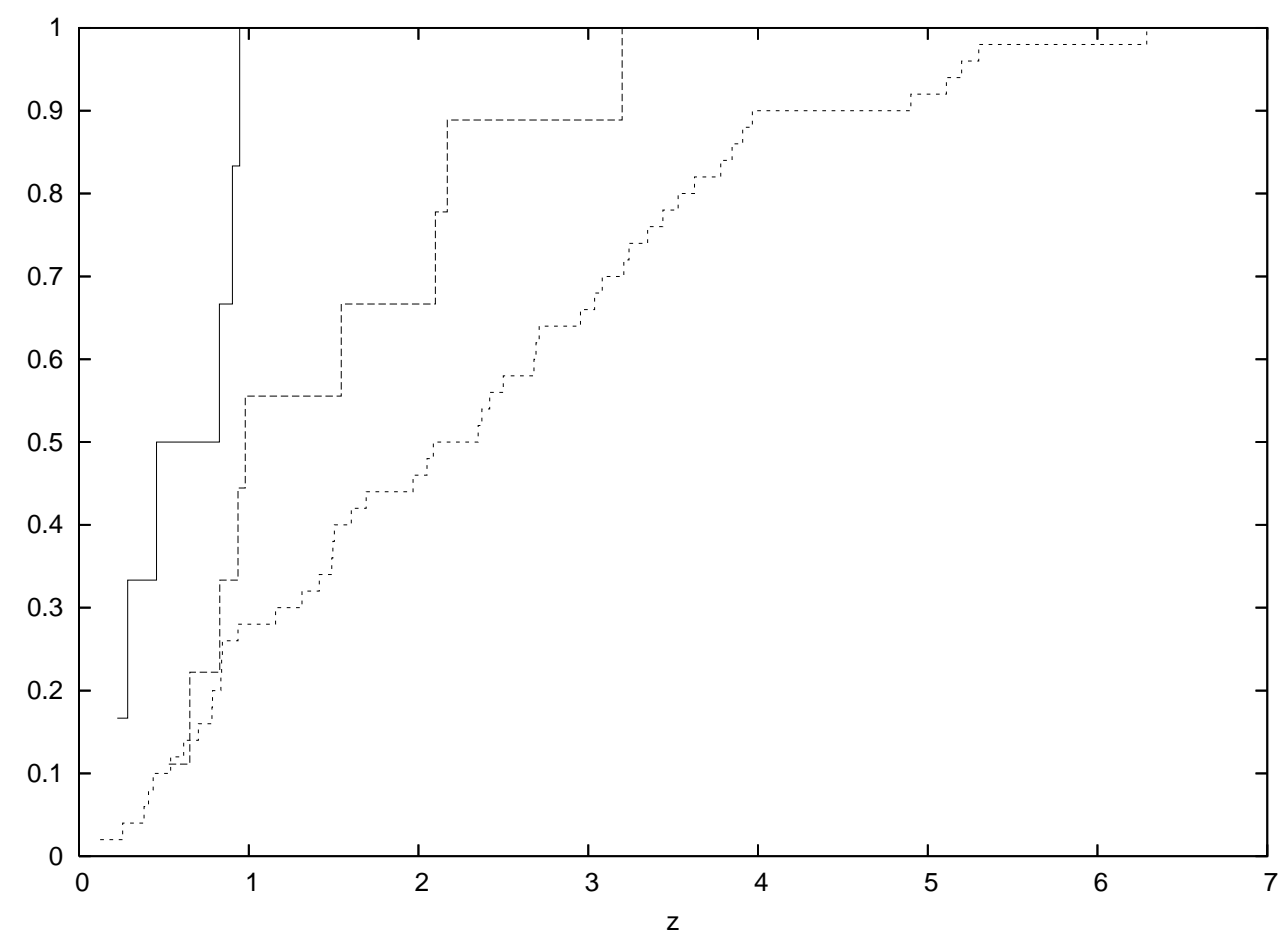

\section{Impact on cosmology}

The cosmological principle requires that the Universe is spatially homogeneous and isotropic on average at scales larger than the size of any structure [20]. In other words, some scale of averaging should exist, which should be larger than any known structure. Observations show that the greatest structures (filaments, voids, superclusters, ...) have sizes at least $\simeq 400 \mathrm{Mpc}$ [21, 22]. Hence, at redshifts $z<0.1$ the matter distribution in the Universe is anisotropic and inhomogeneous, and the scale - where the averaging should be done - should be at least of order $400 \mathrm{Mpc}$.

There are also further observational indications that some structures may exist also at redshift $z<1[23,24,25,26,27]$. They are also supported indirectly by numerical simulations of the spatial distributions [28]. These studies support the existence of structures on the Gpc scale, and challenge the cosmological principle, because the proper-motion distance [29] corresponding to $z=1.0$ is $3.2 \mathrm{Gpc}$ for the most preferred cosmological parameters $\left(\Omega_{M}=0.27, \Omega_{\Lambda}=0.73\right.$ and $H_{o}=71 \mathrm{~km} /(s M p c)$ ). But for the same cosmological parameters the Hubble-radius (measured as proper-motion distance) is $14 \mathrm{Gpc}$, and hence the averaging should be done on a scale between 3.2 and $14 \mathrm{Gpc}$, if the cosmological principle holds. This is artificial but still possible, and also these observations are remarkable but neither decisive. Hence, the cosmological principle is not rejected yet.

The probe of the angular distribution of the gamma-ray bursts is a further observational test of this principle [30], because - if this principle holds - GRBs should be distributed isotropically on 
the sky, if they are dominantly at $z>0.1$. In any case, for this probe GRBs are convenient objects, because they are well seen in the gamma-band also in the Galactic plane.

The detected anisotropy of the BATSE short and intermediate GRBs is in any case remarkable. No direct redshifts from the afterglow measurements are known for these objects. (There is a period in years 1997-2000, when afterglows were already detected, and BATSE was still working. Hence, there are known a few BATSE bursts with directly measured redshifts [for their survey see, e.g., [31]], but all belong to long bursts.) Indirectly it follows that for the short GRBs the redshifts at $z<0.1$ form only a minority and the median is $z \simeq 0.25$ [32]. The redshifts should dominantly be at $0.1<z<1.0[32,33,34]$. Concerning the redshifts of intermediate BATSE bursts the situation is less clear than that of the short bursts, but they can be even at larger redshifts than the short ones [10]. The distribution of the known redshifts of GRBs detected by the Swift satellite are shown on Figure 3.

All this means that the detected anisotropic distribution of the short/intermediate GRBs [30, 33,34 ] gives a further observational challenge of the cosmological principle supporting the earlier papers $[23,24,25,26,27]$.

\section{Conclusions}

The key ideas of this paper can be summarized as follows:

- From the statistical point of view there are three types of GRBs. This conclusion was found for different databases using different statistical tests.

- The short and intermediate GRBs - at least for the BATSE database - are distributed anisotropically on the sky.

- Because these anisotropically distributed objects are dominantly at $z>0.1$, this discovery is a further observational challenge of the cosmological principle.

Acknowledgements. Thanks are due to István Horváth, Péter Mészáros and Roland Vavrek for valuable remarks. This study was supported by the OTKA grant K77795, by OTKA/NKTH A08-77719 and A08-77815 grants (Z.B.), by the Grant Agency of the Czech Republic grant P209/10/0734, by the project SVV 261301 of the Charles University in Prague, and by the Research Program MSM0021620860 of the Ministry of Education of the Czech Republic (A.M. and J. К̌.).

\section{References}

[1] C. Kouveliotou et al. 1993, ApJ, 413, L101

[2] I. Horváth 1998, ApJ, 508, 757

[3] S. Mukherjee et al. 1998, ApJ, 508, 314

[4] I. Horváth 2002, A\&A, 392, 791

[5] I. Horváth et al. 2006, A\&A, 447, 23

[6] A. Balastegui et al. 2001, MNRAS, 328, 283 
[7] T. Chattopadhyay et al. 2007, ApJ, 667, 1017

[8] D. Huja et al. 2009, A\&A, 504, 67

[9] I. Horváth et al. 2008, A\&A, 489, L1

[10] I. Horváth et al. 2010, ApJ, 713, 552

[11] J. Rípa et al. 2009, A\&A, 498, 399

[12] I. Horváth 2009, Ap\&SS, 323, 83

[13] L.G. Balázs 2003, A\&A, 401, 129

[14] P. Veres et al. 2010, ApJ, 725, 1955

[15] L.G. Balázs et al. 1998, A\&A, 339, 1

[16] L.G. Balázs et al. 1999, A\&A Suppl.Ser., 138, 417

[17] A. Mészáros et al. 2000a, ApJ, 539, 98

[18] A. Mészáros et al. 2000b, A\&A, 354, 1

[19] R. Vavrek et al. 2008, MNRAS, 391, 1741

[20] P.J.E. Peebles 1993, Principles of Physical Cosmology, Princeton Univ. Press

[21] M.J. Geller \& J.P. Huchra 1989, Science, 246, 897

[22] J.A. Peacock 2000, Cosmological Physics, Cambridge Univ. Press

[23] C.B. Collins \& S.W. Hawking 1973, MNRAS, 162, 307

[24] P. Birch 1982, Nature, 298, 451

[25] T.J. Broadhurst et al. 1990, Nature, 343, 726

[26] L. Rudnick et al. 2007, ApJ, 671, 40; Erratum: 678, 1531

[27] A. Kashlinsky et al. 2008, ApJ, 686, L49

[28] V. Springel et al. 2006, Nature, 440, 1137

[29] S. Weinberg 1972, Gravitation and Cosmology, J. Wiley and Sons, New York

[30] A. Mészáros et al. 2005, in Proc. IAU Symp. 201, "New Cosmological Data and the Values of the Fundamental Parameters", 7-11 August 2000, Manchester, UK, eds. A. Lasenby \& A. Wilkinson, Astron. Soc. of Pacific, 486

[31] Z. Bagoly et al. 2003, A\&A, 398, 919

[32] Nakar E. 2007, Physics Reports, 442, 166

[33] A. Mészáros et al. 2009a, in AIP Conf. Proc. 1133 "Gamma-Ray Burst: Sixth Huntsville Symposium", eds. C. Meegan et al., 483

[34] A. Mészáros et al. 2009b, Baltic Astronomy, 18, 293 